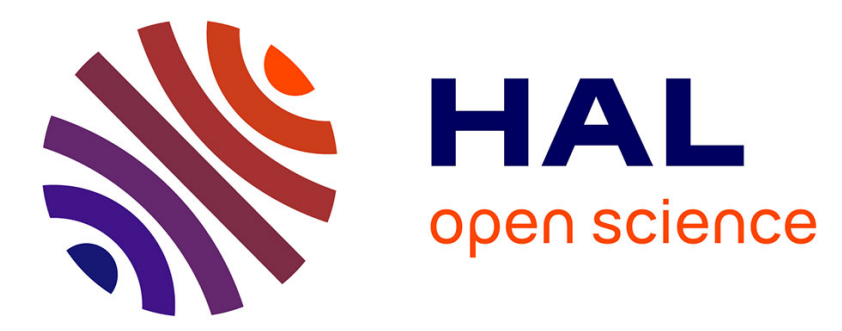

\title{
Investigating Several Wireless Technologies to Build a Heteregeneous Network for the In-Flight Entertainment System inside an Aircraft Cabin
}

\author{
Ahmed Akl, Thierry Gayraud, Pascal Berthou
}

\section{To cite this version:}

Ahmed Akl, Thierry Gayraud, Pascal Berthou. Investigating Several Wireless Technologies to Build a Heteregeneous Network for the In-Flight Entertainment System inside an Aircraft Cabin. Wireless and Mobile Communications (ICWMC), Sep 2010, Spain. pp.532-537. hal-00661013

\section{HAL Id: hal-00661013 https://hal.science/hal-00661013}

Submitted on 18 Jan 2012

HAL is a multi-disciplinary open access archive for the deposit and dissemination of scientific research documents, whether they are published or not. The documents may come from teaching and research institutions in France or abroad, or from public or private research centers.
L'archive ouverte pluridisciplinaire HAL, est destinée au dépôt et à la diffusion de documents scientifiques de niveau recherche, publiés ou non, émanant des établissements d'enseignement et de recherche français ou étrangers, des laboratoires publics ou privés. 


\title{
Investigating Several Wireless Technologies to Build a Heteregeneous Network for the In-Flight Entertainment System Inside an Aircraft Cabin
}

\author{
Ahmed Akl ${ }^{1,2}$, Thierry Gayraud ${ }^{1,2}$, Pascal Berthou ${ }^{1,2}$ \\ ${ }^{1}$ CNRS ; LAAS ; 7 avenue du Colonel Roche, F-31077 Toulouse, France \\ ${ }^{2}$ Université de Toulouse ; UPS, INSA, INP, ISAE ; LAAS ; F-31077 Toulouse, France \\ \{akkl, gayraud, berthou\}@laas.fr
}

\begin{abstract}
A primary difficulty when investigating communication requirements in an aircraft cabin is the diverse needs of passengers when compared to the strict constraints inside the cabin. It is recognized that there is an increasing need of passengers to use their electronic devices as well as the need for entertaining during the flight. This paper aims at integrating heterogeneous available communication technologies, showing their pros and cons -within this contextwhile considering the imposed communication restrictions inside the aircraft's cabin.
\end{abstract}

Keywords - IFE system; WLAN; WUSB; PLC networks

\section{INTRODUCTION}

In recent years, market surveys have revealed a surprising and growing trend in the importance of In-Flight Entertainment (IFE) with regard to choice of airline. With modern long range aircraft the need for "stop-over" has been reduced, so the duration of flights has also been increased. Air flights, especially long distance, may expose passengers (PAX) to discomfort and even stress. IFE can provide stress reduction entertainment services to the passenger. The IFE system is an approach that can utilize the wireless technology for the purpose of exchanging data -in both directions- between PAX and the entertainment system. It can be also used to improve the passenger's service satisfaction level.

As stated by Niebla [1] users are becoming more and more familiar to personal equipments, such as mobile phones, laptops, and PDAs. This shows the importance of providing aircrafts with facilities that support these equipments.

In addition, usage of the wireless technology will help in decreasing the connecting wires; this is a valuable criterion in aircrafts designing. However, using off-shelf technologies inside the cabin is usually not applicable when using them in the usual manner; the environment inside the cabin has very strict constrains since safety is a major requirement. Consequently, using just one technology can't be the optimum solution. In fact, using a combination of different technologies can provide a better service while overcoming the existing constrains. How to allow PAX to use their Personal Electronic Devices (PED) (i.e., mobile phones, laptop, etc.) was usually done through specialized devices [1][2]. Nevertheless, there is no current research to use a combination of off-shelf technologies inside the cabin.

In the next section we will introduce an overview for an IFE system showing its components and requirements.
Section III introduces the communication challenges that face the IFE systems. Section IV shows the proposed available technologies that can be used to overcome the challenges in such environment. Section V introduces our approach for integrating them together.

\section{BACKGROUND AND RELEVANT WORK}

The recognized economics of wireless networks and communications systems have made them an attractive target for environments where individual wires are cumbersome. An airplane cabin is such an environment. Dwayne [3] said that due to the need of rapidly reconfiguring the cabin's seating, it is further assumed that wireless networking, rather than cable or fiber optics, must be used to interconnect passenger's entertainment equipment with other elements of the system.

The use of wireless communication technologies on board of an aircraft provides an opportunity to remove wiring and save weight on the aircraft. The weight savings can be directly measured in terms of fuel savings and improved operating economics over the life time of an aircraft. However, there is a need to ensure that there is no interference with the aircraft's communication and navigation systems.

\section{A. The need for IFE systems}

Hao [4] mentioned that the enclosed environment of the aircraft can cause discomfort or even problems to PAX. IFE systems can greatly reduce these negative effects. This can be done by using e-books, video/audio broadcasting, games, internet, and On Demand services. The fact that the PAX come from highly heterogeneous pools (such as age, gender, ethnicity, etc...) causes an impact on the adaptive interface systems.

As mentioned by Fariba [5], the IFE systems usually include screen-based, audio and communication systems. The screen-based products include video systems enabling PAX to watch movies, news and sports. These systems had progressed into video-on-demand, allowing PAX to have control when they watch movie. Air map display is another service that allows PAX to locate their flight's route Exterior-view cameras also enable PAX to have the pilot's forward view on take-off and landing on their personal TV screens. Audio systems include different types of music channels and special programs recorded for the airlines. Communication systems include intra-communications with devices such as telephones, facsimile and in-seat power 
supplies, and inter-communications between the screenbased system and its subsystems (i.e., remote control).

\section{B. The IFE system's components}

In fact, the entertainment starts from the passenger's seat design where most of the IFE system components are embedded. Wiring cables connect together all of the electronic devices in the seat as well as connecting them to the whole system in the cabin. They run through the cabin's walls, floor, and seats. Unfortunately, conveying signals and power to the seats with a connector for each seat would cause reliability and maintenance problems, and hinder timely cabin reconfiguration.

Nowadays, IFE systems are interactive systems, so a Passenger Control Unit (PCU) is usually needed to control the surrounding devices. The PCU should be compact and easily held. Moreover, the pocket holding the PCU has to be placed in a way that makes it easily reached and not to affect the passenger's comfort.

A Seat Electronic Box (SEB) can be used to connect the system's different components together. It is used to connect the passenger's devices and the IFE system instead of having a separate channel for each signal. For example, to transmit communication and video signals, two different networks should be available if the SEB is not used. When using the $\mathrm{SEB}$, the communication and video devices are connected directly to it to convey signals to the rest of the IFE system through one single network. Then, it simplifies and facilitates maintenance procedure since malfunctioning devices can be easily replaced without affecting the IFE connections.

Halid [6] stated that PLCs can provide a way of communication through power lines networks. Power lines and communication networks have different physical characteristics, so a PLC modem must be used as an interface between the two networks. They must be designed to provide accepted network operation under typical power lines transmission conditions. However, power lines are not designed as a good transmission media. It suffers from attenuation, fading, and noise. Nevertheless, the great advances in digital signal processing, error detection and correction, modulation, media access control techniques encourage the use of PLC in communication field.

\section{COMMUNICATION CHALLENGES}

Although aircraft security may be seen as another burden due to its very strict requirements, but it is mandatory to be included during the design of communication and data services. A major concern for using wireless devices in aircraft cabin is their interference with the aircraft's communication and navigation system, especially unintended interference from PAX's Personal Electronic Devices (PED). Holzbock [7] said that the installed navigation and communication systems on the aircraft are designed to be sensitive to electromagnetic signals, so they can be protected against PAX's emitters by means of frequency separation. In addition, Jahn [2] mentioned that there are two types of PEDs' interference, intentional and spurious. The former is the emissions used to transmit data over the PED's allocated frequency band. The latter is the emissions due to the RF noise level.

Moreover, the existing systems suffer from bandwidth limitations; the trend toward bandwidth-consuming Internet services currently cannot be satisfied [2]. PAX number and categories can be considered as a factor that affects network scalability. For example, the network bandwidth should be increased if the number of the first class PAX was increased to support the increasing need for video stream.

It is stated by Holzbock [7] that existing indoor channel models mainly investigate office or home environments, thus these models may not be appropriate for modeling an aircraft cabin channel. Attenuation of walls and multi path effects in a 'normal' indoor environment are effects which are not expected to be comparable to the effect of the higher obstacle density in a metallic 'tunnel'. The elongated structure of a cabin causes smaller losses, than that expected in other type of room shapes. However, the power addition of local signal paths can lead to fading of the signal in particular points. In addition, small movements of the receiver can have a substantial effect on reception. The same opinion was emphasized by Diaz [8]. To overcome this problem, Youssef [9] used the commercial software package Wireless Insite to model the electromagnetic propagation of different wireless access points inside different types of aircrafts. Another challenge is that the cabin of an aircraft and the aeronautical environment in general define a very specific scenario that presents several constraints which will affect the coverage and capacity planning. This is due to the fact that the space is very limited in an aircraft cabin, and its design allows installing equipment only in specific locations, where the configuration of the panels is easy to disassemble for maintenance [1]. Therefore, the replacement technique associated with the IFE system components, may affect the welling of the companies to use them. Replacing time consuming parts can lead to a long aircraft downtime or flight delays. Also, a device that fails during the flight, and is difficult to be replaced, will cause the passenger to be unsatisfied. Consequently, it is advisable to design components that are easily replaced with the minimum required technical skill.

\section{PROPOSED COMMUNICATION TECHNOLOGIES}

As mentioned by Holzbock [7], wireless Cabin aims at developing a communication infrastructure consisting of heterogeneous wireless access networks to provide aircraft PAX and crew members with access to IFE system. PAX are able to access different services through state-of-the-art wireless access technologies such as W-LAN IEEE802.11, and Bluetooth.

\section{A. Ethernet}

Ethernet is currently the standard for wired communication in different fields. Haydn [10] showed that it is characterized by interesting features such as good communication performance, scalability, high availability, and resistance to external noise. However, Ethernet cabling is considered a burden for aircraft design 


\section{B. Wireless $L A N$}

WLAN is a well known technology used in different commercial, industrial, and home devices, and can easily coexist with other technologies to form a heterogeneous network [1]. Jim [11] stated that WLAN and Bluetooth technologies are too complementary not a competing technologies. They can cooperate together to provide users with different connecting services.

\section{Wireless USB}

USB technology allows different peripherals to be connected to the same PC more easily and efficiently than other technologies such as serial and parallel ports. However, cables are still needed to connect the devices. This raised the issue of Wireless USB (WUSB) where the devices can have the same connectivity through a wireless technology. Neal [12] stated that although it is difficult to achieve a wireless performance similar to wired USB, but the rapid improvements in radio communication can make WUSB a competent rival. It is based on the Ultra Wide Band (UWB) technology. In Europe, it supports a frequency range from 3.1 to $4.8 \mathrm{GHz}$. Moreover, Udar [13] mentioned that UWB communication is suitable for short range communications which can be extended by the use of mesh networks. Although WUSB was designed to satisfy client needs, but it can also be used in a data centre environment. He discussed how WUSB characteristics can match such environment. This application can be of a great help in IFE systems which strive to massive data communication to support multimedia services and minimizing the connection cables. Moreover, Jong [14] discussed the design issues related to WUSB. He stated that WUSB can support up to $480 \mathrm{Mbps}$, but in real world it doesn't give the promised values; and he showed the effect of design parameters on the device's performance.

\section{Power Line Communication}

A PLC network can be used to convey data signals over cables dedicated to carry electrical power; where PLC modems are used to convert data from the digital signal level to the high power level; and vice versa. Using an existing wiring infrastructure can dramatically reduce costs and effort for setting up a communication network. Moreover, it can decrease the time needed for reconfiguring the cabin's layout since less cables are going to be relocated.

However, such technology suffers from different problems. A power line cable works as an antenna that can produce Electromagnetic Emissions (EME). Thus, the PLC device must be Electromagnetic Compatible (EMC) to the surrounding environment. This means that it must not produce intolerable EME, and not to be susceptible to them. To overcome this problem, the transmission power shouldn't be high in order not to disturb other communicating devices [6]. However, Working on a limited power signal makes the system sensitive for external noise. In spite of this, the PLC devices can work without concerns of external interference due to two reasons. Firstly, the PLC is divided into segments; this minimizes signal attenuation. Secondly, all the cabin's devices are designed according to strict rules that prevent EME high enough to interfere with the surrounding devices.

\section{A HETEROGENEOUS ARCHITECTURE}

IFE system is a field starving for unusual ideas. PAX can be satisfied by receiving services dedicated to a single user, but it will be more amusing if they can be offered services for multiple users, where PAX of similar interests can share their time. Using a single communication technology inside the cabin can't yield satisfactory results, but a combination of different technologies can have a great impact on the provided services.

The term heterogeneous in the networking domain usually implies the mix between wireless and wired networks. However, we mean by heterogeneity, the existence of different networking technologies cooperating together to achieve certain services. The network can be divided into User Technology and System Technology. A User Technology is the technology apparent and directly used by the user (i.e., Bluetooth, WiFi, etc...) to connect his devices to the system. A System Technology is the technology used by the system and is hidden from the user (i.e., PLC).

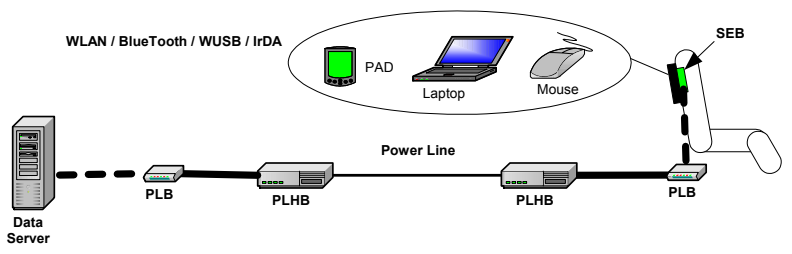

Figure 1. Heterogeneous network architecture

In our proposed heterogeneous architecture, we suggest the usage of a PLC network to convey data between a data server and the PAX seats where he use his PEDs. In addition, wireless APs are connected to the PLC backbone as well; while WUSB is used to provide a way to connect some USB devices to the network (see Figure 1).

\section{A. $P L C$}

In this section, we will introduce some experimentation results to show the applicability of using PLCs for a cabin's IFE system. As shown in Figure 1, the PLC system consists of a Power Line Head Box (PLHB) and a Power Line Box (PLB). The PLHB connects the two terminals of the power line to connect the data server with the seats. Each PLHB service a group of seats which are equipped with PLB per seat. The PLB is responsible for distributing the signal received by the PLHB to the seat's SEB. Each PLHB can support up to $20 \mathrm{PLBs}$ at a rate of $3480 \mathrm{bit} / \mathrm{sec}$. Both PLHB and PLB devices can be configured through their internal web interface to define their IP address and other configuration parameters.

The MGEN (version 4.2) [15] traffic generator was used to emulate the traffic produced by the data server, and a laptop was used as a substitute to the SEBs. The target of the test was to collect different statistics to study the behavior of the PLC system by injecting periodic traffic flows at constant intervals. 


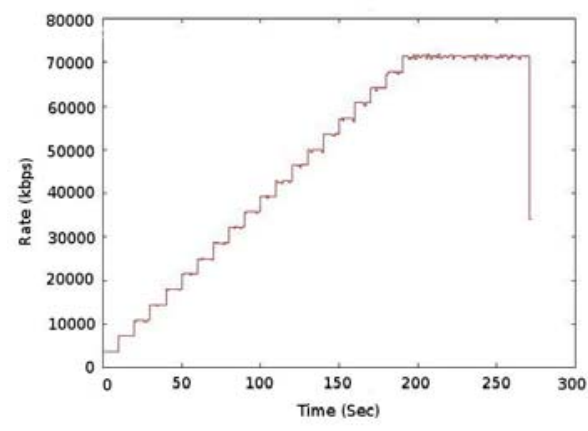

Figure 2. Flow rate of all flows

Figure 2 shows the sum of flows' rates. The stepping of the flow rate is constant indicating that the PLC connection is able to carry the 20 flows. In addition, Figure 4 represents the packet count of the first flow. It is clear that the packet count stayed constant from the start to the end of the simulation without being affected by the injection of the subsequent flows. This emphasis the same results derived from Figure 2

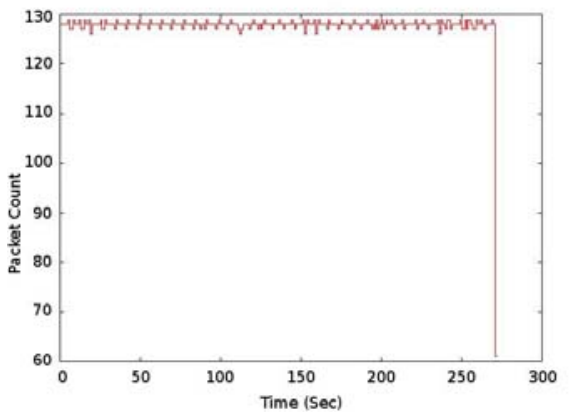

Figure 3. Packet count of the first flow

However, it is normal to have packet dropping during transmission; this is illustrated in Figure 3 showing the obtained loss fraction. It is less than 0.05 which can be considered as a good value. Such configuration can provide the IFE with a way to provide video services by using the existing power cabling.

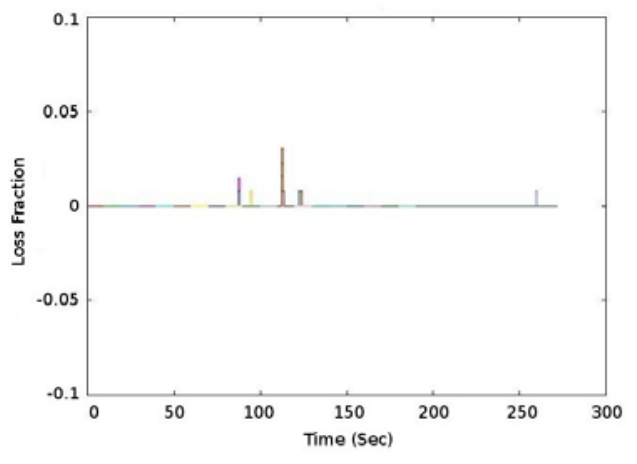

Figure 4. Loss Fraction

\section{B. WLAN}

We held different NS2 [16] simulations to propose a good distribution for the wireless Access Points (APs) inside the cabin. We used the same cabin configuration used by Alexandaros [17]. The cabin consists of 26 rows with 6 seats each ( 3 on each side of the aisle); this gives a total of 156 seats. The cabin is $21 \mathrm{~m}$ long and $3.54 \mathrm{~m}$ wide. The rows' separation distance is $81 \mathrm{~cm}$.

A wireless node - representing a passenger's device - is located at the position of each seat, and APs are used to connect them with the data server. Using large number of wireless devices in a very narrow metallic tunnel like the cabin has a dramatic effect on the network's performance. For this reason, we are studying the effect of using frequency separation between APs. However, we need to determine the minimum number of APs required to cover the whole cabin, and their distribution inside the cabin, so we experimented with three scenarios. In scenario 'A', all nodes (each has a transmission range covering the whole cabin) are using the same communication channel. Scenario 'B' uses nodes with short transmission range, which allows connection only to the nearest Access Point (AP), while using the same channel. Scenario ' $\mathrm{C}$ ' shows nodes with short transmission range and using channel separation. The channel separation in the third scenario is based on the fact that 802.11 only allows the usage of three non interfering channels (i.e., channels 1, 6 , and 11). The impact of the three scenarios over average throughput, average delay, and number of transmitted packets is studied.

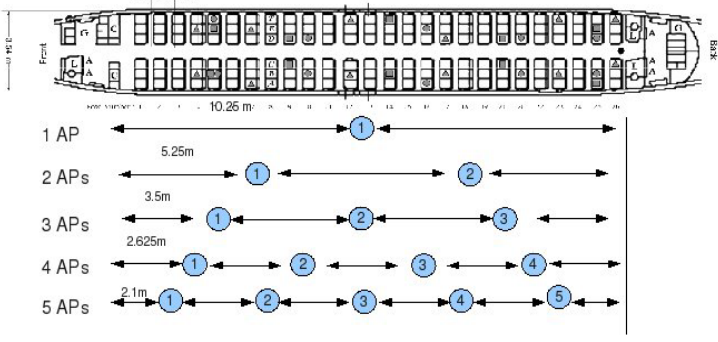

Figure 5. APs distribution

Each scenario was repeated 5 times while using different numbers of Aps located at the aisle. We started by using one AP and the number is incremented until we reached the maximum number of APs which was determined according to the cabin's dimensions. The AP's transmission power was adjusted to minimize the transmission range, so the signal can travel a distance just enough to reach the seat beside the window in order to minimize the effect of its reflection. This allowed us to use a maximum number of 5 APs (Figure 5).

For all scenarios, the nodes (156 node + APs) were configured to have a large queue that can hold up to 1000 packets in order to prevent packet dropping. The transmission power was adjusted to $10 \mathrm{~mW}$ as the minimum value defined in the 802.11 standard. In scenarios that use different channels, Channels 1, 6, and 11 were adjusted to their frequencies $2.412 \mathrm{e} 9 \mathrm{~Hz}, 2.437 \mathrm{e} 9 \mathrm{~Hz}$, and $2.462 \mathrm{e} 9 \mathrm{~Hz}$ respectively. The Rx threshold was determined according to the required transmission range. It was calculated by the tool 
"Threshold" provided as a separate program with the NS2 simulator. TABLE I. shows the values used with each number of APs. For each simulation, the APs were distributed evenly throughout the aisle to provide a full coverage for the cabin.

\section{TABLE I. RX THRESHOLD VALUES}

\begin{tabular}{|c|c|c|}
\hline Number of APs & $\begin{array}{c}\text { Transmission } \\
\text { range(meters) }\end{array}$ & Rx threshold \\
\hline 1 & 10.5 & $8.97474 \mathrm{e}-9$ \\
\hline 2 & 5.25 & $3.58989 \mathrm{e}-8$ \\
\hline 3 & 3.5 & $8.07726 \mathrm{e}-8$ \\
\hline 4 & 2.625 & $1.43596 \mathrm{e}-7$ \\
\hline 5 & 2.1 & $2.24368 \mathrm{e}-7$ \\
\hline
\end{tabular}

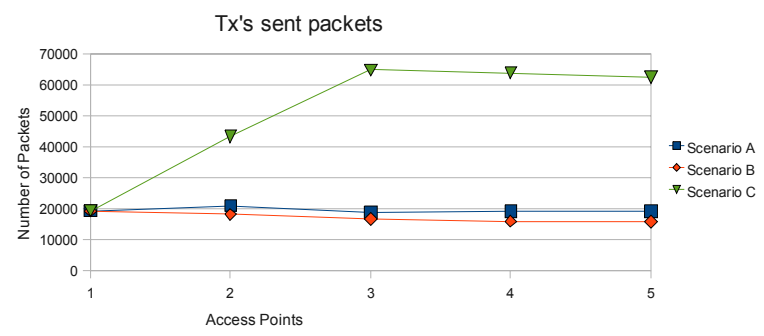

Figure 6. Packets sent by the transmitter

When comparing the three scenarios we can find that using just different number of APs doesn't have a great impact on the networks performance, but when accompanied with channel separation the networks performance is dramatically enhanced. Figure 6, Figure 7, and Figure 8 combine the results of scenarios $\mathrm{A}, \mathrm{B}$, and $\mathrm{C}$. It is noticeable that there is no great difference between scenario $\mathrm{A}$ and $\mathrm{B}$; this is due to the existence of large number of nodes in a small area. In addition, there are many nodes in the shared zone between every two APs. In this zone, nodes are able to detect two APs, but they select just one of them. In other words, on the physical level signals are interfering, while on the logical level only one AP is seen. However, as the number of APs increase, the difference between scenario A and B starts to increase slightly; this is because the number of nodes in the shared zone becomes less, so the interference decreases. On the contrary, when using channel separation (i.e., scenario C) performance was dramatically enhanced after using 3 APs.

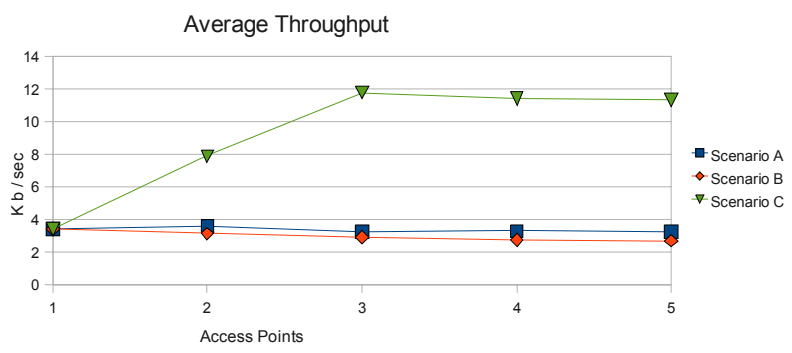

Figure 7. Average Throughput
It is worthy to note that the number of nodes assigned to each AP affects its performance; the fewer nodes we use, the higher performance we get. When using 1, 2, 3, 4, and 5 APs, each AP will have 156, 78, 52, 39, and 32 nodes respectively. However, the difference in the number of assigned nodes with 3,4 , and 5 APs is small. This justifies the reason for saturation after using more than 3 APs; where APs almost handles the same amount of APs

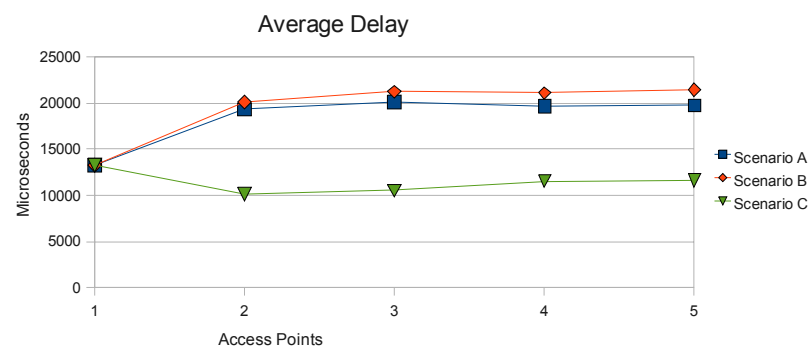

Figure 8. Average delay

\section{WUSB}

Using WUSB to connect passenger's devices seems to be an appealing solution since it doesn't require any additional adapters or connectors, and avoids interference with other wireless technologies (i.e., WLAN, Bluetooth, etc...) by using different bandwidth.

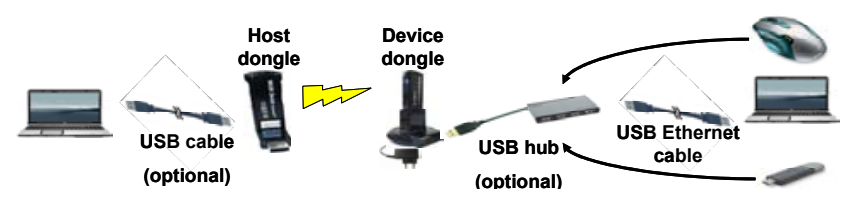

Figure 9. WUSB test-bed

Figure 9 shows our WUSB experimentations test-bed. WUSB Host and Device dongles were used to connect USB devices. The Host dongle is connected to the computer USB port, while the Device dongle connects the USB devices. The dongles driver allows changing of transmission as well as the transmission channels.

1) Connecting different USB devices

Connecting multiple USB devices (i.e., mouse, and keyboard) was done in two different ways; firstly by using two Device dongles for each USB device, secondly by using a USB hub. The results of the first approach were not satisfactory because the two dongles were using the same channel causing interference between them. However, the Host dongle has the ability to choose between seven different channels. In other words, it is possible to use seven Hosts at the same transmission range without any interference between them. The second approach gave better performance. Moreover, a hub is much more economical than using a WUSB dongle dedicated for each device. 


\section{2) File transfer}

It is important to know if WUSB is capable of transferring large files, and to what extent it is comparable to wired USB, so 4064 files of size $892 \mathrm{MB}$ were transferred to a flash USB storage device using WUSB and wired USB. The results shown in TABLE II. indicate that WUSB are slower by almost $60 \%$ than wired USB.

TABLE II. WUSB VS WIRED USB

\begin{tabular}{|c|c|c|}
\hline & Elapsed time & Delay ratio \\
\hline WUSB & $915 \mathrm{secs}$ & $((344 / 915) * 100)-100$ \\
\hline Wired USB & $344 \mathrm{secs}$ & $=62.4 \%$ \\
\hline
\end{tabular}

\section{3) Transmission range with different power levels}

The test started by putting the Host dongle and the Device dongle on the same line of sight; then the device dongle is moved away until it is disconnected. The same procedure was repeated while using two Device dongles. The two dongles are placed at the same horizontal level with a separation of few centimeters, and are moved together. The whole experiment was repeated while changing the dongles transmission power level (i.e., low, normal and strong).

TABLE III. TRANSMISSION RANGE

\begin{tabular}{|c|c|c|}
\hline \multirow{2}{*}{$\begin{array}{c}\text { Transmission } \\
\text { power }\end{array}$} & Max. achieved distance (meters) between Tx and Rx(s) \\
\cline { 2 - 3 } & Single device & Dual device \\
\hline Low & 7 & 4.2 \\
\hline Normal & 12 & 6.3 \\
\hline Strong & 16 & 8.4 \\
\hline
\end{tabular}

As shown in TABLE III. , the existence of two dongles at the same area, and working at the same channel has a dramatic effect on transmission range, so when considering that the distances between seats inside the cabin is considerably short when compared with the minimum transmission range, then it is highly recommended to use different channels for neighboring dongles.

\section{CONCLUSION AND FUTURE WORK}

Heterogeneous network architecture is a promising solution for such application. Using PLC networks can be a competitive solution since it decreases the amount of cabling inside the cabin, and can be used to connect the APs (to support mobility) directly to the network system. Moreover, it overcomes the interference constrain, and can provide enough bandwidth to support heavy traffic required for multimedia services. When combined with WUSB, it becomes easier for PAX to connect their PEDs.

Through experimentation results and simulations, this work proves that it is possible to build a heterogeneous network which contains all the mentioned technology; each to solve a certain part of the problem. Multimedia content distribution supported by PLC and Ethernet architecture added to personal communication supported by WUSB can provide the IFE system with a satisfactory solution needed for such systems. This can be done without interfering with each other.

It is planned to investigate the feasibility of using other technologies such as UWB and smart antennas; where low energy transmissions with high bandwidth while covering certain regions can be used to increase performance. In addition, scalability issues of WUSB need more investigation.

\section{REFERENCES}

[1] C. Niebla, "Coverage and capacity planning for aircraft in-cabin wireless heterogeneous networks," IEEE Vehicular Technology Conference, 2003, pp. 1658-1662

[2] A. Jahn and M. Holzbock, "Evolution of aeronautical communications for personal and multimedia services," IEEE Communications Magazine, vol. 41, 2003, pp. 36-43.

[3] D. Folden, T. Jackson, M. Panique, R. Tiensvold, R.S. Wolff, T. Howard, E. Julian, L. Junkert, D. Lopez, and M.J. Oudshoorn, "An Aircraft Cabin Wireless System for Games and Video Entertainment," ACM Computer in Entertainment, 2007.

[4] H. Liu, "In-Flight Entertainment System: State of the Art and Research Directions," Second International Workshop on Semantic Media Adaptation and Personalization (SMAP 2007), Second International Workshop on Semantic Media Adaptation and Personalization (SMAP 2007), 2007, pp. 241-244.

[5] F. Alamdari, "Airline in-flight entertainment: the passengers' perspective," Journal of Air Transport Management, vol. 5, 1999, pp. 203-209.

[6] H. Hrasnica, A. Haidine, and R. Lehnert, Broadband Powerline Communications Networks, John Wiley \& Sons, Ltd, 2004.

[7] M. Holzbock, Y. Hu, A. Jahn, and M. Werner, "Advances of aeronautical communications in the EU framework," International Journal of Satellite Communications and Networking, vol. 22, 2004, pp. 113-137.

[8] N.R. Diaz and J.E. Esquitino, "Wideband Channel Characterization for Wireless Communications inside a short haul aircraft," Vehicular Technology Conference, 2004.

[9] M. Youssef, L. Vahala, and J. Beggs, "Wireless network simulation in aircraft cabins," IEEE Antennas and Propagation Society Symposium, vol. 3, 2004, pp. 2223-2226.

[10] H. Thompson, "Wireless and Internet communications technologies for monitoring and control," Control Engineering Practice, vol. 12, 2004, pp. 781-791.

[11] J. Lansford, A. Stephens, and R. Nevo, "Wi-Fi (802.11b) and Bluetooth: Enabling Coexistence," IEEE Communications Magazine, 2001, pp. 20-27.

[12] N. Leavitt, "For Wireless USB, the Future Starts Now," IEEE Computer Society, vol. 40, 2007, pp. 14-16.

[13] N. Udar, K. Kant, R. Viswanathan, and D. Cheung, "Characterization of Ultra Wide Band Communications in Data Center Environments," Proc. of ICUWB, 2007.

[14] J. M. Sohn, S. H. Baek, and J. D. Huh, "Design issues towards a high performance wireless USB device," IEEE International Conference on Ultra-Wideband, vol. 3, 2008, pp. 109-112.

[15] MGEN site, http://pf.itd.nrl.navy.mil/mgen/mgen.html (Last visit 5/7/2010)

[16] NS2 site:, http://www.isi.edu/nsnam/ns/ (Last visit 5/7/2010)

[17] A. Kaouris, M. Zaras, M. Revithi, N. Moraitis, and P. Constantinou, "Propagation Measurements inside a B737 Aircraft for In-Cabin Wireless Networks," VTC Spring 2008 - IEEE Vehicular Technology Conference, IEEE, 2008, pp. 2932-2936. 